\title{
The impact of curcumin derived polyphenols on the structure and flexibility COVID-19 main protease binding pocket: $A$ molecular dynamics simulation study
}

\author{
Aweke Mulu ${ }^{\text {Corresp., } 1}$, Mulugeta Gajaa ${ }^{2}$, Haregewoin Bezu Woldekidan ${ }^{1}$, Jerusalem Fekadu W/mariam ${ }^{1}$ \\ ${ }^{1}$ College of APPLIED SCIENCE, ADDIS ABABA SCIENCE AND TECHNOLOGY, ADDIS ABABA, Non-US/Non-Canadian, Ethiopia \\ 2 College of Natural and Social science, ADDIS ABABA SCIENCE AND TECHNOLOGY, ADDIS ABABA, Non-US/Non-Canadian, Ethiopia \\ Corresponding Author: Aweke Mulu \\ Email address: aweke.mulu@aastu.edu.et
}

The newly occurred SARS-CoV-2 caused a leading pandemic of coronavirus disease (COVID-19). Up to know it has been infected more than one hundred sixty million and killed more than three million people according to 14 May 2021 World Health Organization report. So far, different types of studies have been conducted to develop anti-viral drug for COVID-19 with no success yet. As part of this, silico studies used to discover and introduce COVID-19 antiviral drugs and results showed that protease inhibitors could be very effective in controlling. The aim of this study is to investigate the binding affinity of three curcumin derived polyphenols against COVID-19 the main protease (Mpro), binding pocket and identification of important residues for interaction. In this study, molecular modeling, auto-dock coupled with molecular dynamics simulations was performed to analyze the conformational and stability of COVID-19 binding pocket with diferuloylmethane, demethoxycurcumin and bisdemethoxycurcumin. All three compounds has showed binding affinity $-39,-89$ and -169.7 , respectively. Demethoxycurcumin and bisdemethoxycurcumin showed optimum binding affinity with target molecule and these could be one of potential ligands for COVID-19 therapy. And also, COVID-19 main protease binding pocket binds with the interface region by one hydrogen bond. Moreover, the MD simulation parameters indicated that demethoxycurcumin and bisdemethoxycurcumin were stable during the simulation run. These findings can be used as base line to develop therapeutics with curcumin derived polyphenols against COVID-19. 
1 The impact of curcumin derived polyphenols on the structure and flexibility 2 COVID-19 main protease binding pocket: A molecular dynamics simulation study

3 Aweke Mulu Belachew ${ }^{1 *}$, Mulugeta Gajaa Ufgaa $^{2}$, Haregewoin Bezu Woldekidan ${ }^{3}$ and

4 Jerusalem Fekadu W/ Mariam ${ }^{4}$

$5 \quad$ 1,3,4 College of Applied Science, Addis Ababa Science and Technology University, Addis Ababa, 6 Ethiopia

$7 \quad{ }^{2}$ College of Natural and Social science, Addis Ababa Science and Technology University, Addis

8 Ababa, Ethiopia

9 Corresponding Author:

10 Aweke Mulu Belachew ${ }^{1 *}$

11 Addis Ababa, Ethiopia, 16417, Ethiopia

12 Email address: aweke.mulu@aastu.edu.et

\section{Abstract}

14 The newly occurred SARS-CoV-2 caused a leading pandemic of coronavirus disease (COVID15 19). Up to know it has been infected more than one hundred sixty million and killed more than three million people according to 14 May 2021 World Health Organization report. So far, different types of studies have been conducted to develop anti-viral drug for COVID-19 with no success yet. As part of this, silico studies used to discover and introduce COVID-19 antiviral drugs and results showed that protease inhibitors could be very effective in controlling. The aim of this study is to investigate the binding affinity of three curcumin derived polyphenols against COVID-19 the main protease (Mpro), binding pocket and identification of important residues for interaction. In this study, molecular modeling, auto-dock coupled with molecular dynamics simulations was performed to analyze the conformational and stability of COVID-19 binding pocket with diferuloylmethane, demethoxycurcumin and bisdemethoxycurcumin. All three compounds has showed binding affinity $-39,-89$ and -169.7 , respectively. Demethoxycurcumin and bisdemethoxycurcumin showed optimum binding affinity with target molecule and these could be one of potential ligands for COVID-19 therapy. And also, COVID-19 main protease binding pocket binds with the interface region by one hydrogen bond. Moreover, the MD simulation parameters indicated that demethoxycurcumin and bisdemethoxycurcumin were stable during the simulation run. These findings can be used as base line to develop therapeutics with curcumin derived polyphenols against COVID-19.

Keywords: NAMD; X-ray crustal structure; SARS-CoV-2; Docking; binding energy 


\section{Introduction}

34 The severe acute respiratory syndrome coronavirus 2 (SARS-CoV-2), which was first reported in 35 Hubei Province of Wuhan, China in December 2019, is responsible for the ongoing global 36 pandemic of coronavirus disease 2019 (COVID-19) spreading across almost all countries with 160686749 active infection cases and 3335948 deaths until 14 May 2021 [1 2 10]. The actual number of cases presumed much higher, due to limitation in testing, lack of medication, and applying World Health Organization recommendation such as, social distancing, hand washing, and travel ban and full lockdown in many cities [ $\left[\begin{array}{lll}1 & 3 & 10\end{array}\right]$. Prior work in this area showed that - SARS-CoV-2 is the third coronavirus belonging to Genus Beta coronavirus that can infect human next coronavirus-severe acute respiratory syndrome (SARS-CoV-2) and the Middle East respiratory syndrome (MERS-CoV) [2]. SARS-CoV-2, the virus responsible for COVID-19, belongs to a group of genetically related viruses that includes SARS-COV and a number of other CoVs isolated from bat populations [1]. The SARS-CoV-2 virus is the primary causative agent of COVID-19. The pol gene of the SARS-CoV-2 virus possesses a positive-sense $\sim 30 \mathrm{~kb}$ long RNA genome with $\sim 14$ ORFs and encoding 27 proteins [3 39]. Among multiple encoded proteins Programmed ribosomal frame shifting generates two polyproteins encoding the replicase proteins [4]. Range of viral processing protein associated with structural proteins, non-structural proteins, and accessory proteins have been reported in several studies [ 14 5]. The pol gene of the SARS-CoV-2 virus encodes two protease enzymes main protease (Mpro) and Papain-like protease, which are involved in the proteolytic processing of the polyproteins into individual nsp7/nsp8/nsp12 to control viral gene expression and replication in the host [ 34 5 $]$. SARS-CoV2 virus Mpro is a vital enzyme that catalyzes the proteolytic process, non-structural proteins (nsps) generated by the main protease play a major role in the reverse-transcribed viral DNA into the host genome [ $\left.\begin{array}{llll}4 & 6 & 4 & 45\end{array}\right]$. It is composed of three structurally and functionally distinct domains: domain I, II, and III. Domain I and II contain highly conserved Cys145-His41 residues located in the cleft which are directly involved in the catalytic activities of Mpro [7]. Therefore, SARS-CoV-2 virus Mpro has emerged as a promising antiviral target as this is responsible for newly synthesized double-stranded viral DNA and subsequent maturation of polyproteins into host genomic DNA. SARS-CoV-2 virus Mpro inhibitors repurposing, which target the enzyme active site, have been studied computationally over the past few months to control COVID-19 as there is an urgent requirement for a strong drug or combination of drugs to combat the pandemic 
64 [8 9]. Currently, natural product are screened by molecular docking and molecular dynamic 65 simulation to test their affinity towards molecular targets of COVID-19 taking the advantage that

66

67

68

69

70

71

72 73

74

75

76

77

78

79

80

81

82

83

84

85

86

87

88

89

90

91

92

93

94 natural products are free from toxic or side effects [44 46 47]. In this study, the structure of SARS-CoV-2 virus Mpro was obtained from the protein data bank (PDB) gave a major advance for structure-based drug design of Curcumin derivative polyphenols, and gives well-established techniques to reveal important dynamic information in proteins. This is an exciting new approach to develop effective therapies to fight COVID-19 with Curcumin, exploiting a new mechanism based on well-validated virulence factors. And also, expanding the list of lead compounds means more possible drugs that could be advanced through clinical trials and better treatments will be possible shortly.

Up to now, there are no robust drugs for the wide-spread SARS-CoV-2 virus although there are now several vaccines that are in use. Finding a new drug in a wet lab and bringing it to market is hard, expensive, and time-consuming. Due to rapid ongoing global health emergency in the current outbreak and high mortality rate estimated by World Health Organization, the more rapid development of new antiviral drugs is highly demanded [10 39]. In the literature, traditional medicines have been extensively investigated to find novel therapeutic strategies for viruses including SARS-CoV-2. Evidence from several computational studies indicated that natural compounds for virus infection treatment play a critical role. A review of the literature showed that natural compounds with low cytotoxicity and high bioavailability seem to be the most efficient candidate's therapy. And also, studies observed lining modern medicine, humans have relayed to the use of phytochemicals for the treatment of different diseases [ $\left[\begin{array}{ll}11 & 12\end{array}\right]$. In general work to date in this area supports phytochemicals such as polyphenols possessing a variety of potential biological benefits such as Anti-oxidant, Anti-inflammatory, antiviral, and antibacterial [13 14]. A systematic review of peer-reviewed literature showed that Curcumin has antiviral activity in human immunodeficiency virus, herpes simplex virus, dengue virus, Zika, and chikungunya viruses [ $\left[\begin{array}{llll}13 & 14 & 15\end{array}\right]$. The main objective of this work is to investigate methods for improving Curcumin derivative polyphenols that will be required for developing Mpro inhibitors were performed with silico study that included molecular docking combined with molecular dynamic simulation depend on Mpro binding pocket (PDB ID: 7BUY) [7]. Specifically, we aim to investigate the amino acids that contribute the most to the binding Curcumin-derived polyphenols; we examined the binding mode of the Mpro pocket and found that form hydrogen 
95 bonds with binding pocket of residues. Finally, we put into MD simulation and binding free 96 energy calculation. Results revealed that demethoxycurcumin and bisdemethoxycurcumin 97 (especially bisdemethoxycurcumin), could have good inhibitory activity towards Mpro.

\section{Materials \& Methods}

99 Protein and Polyphenols Structure Preparation

100 The crystal structure of the MPro: carmofur complex (PDB ID: 7BUY) [7] was retrieved from 101 the Protein Data Bank (PDB). A high-quality homology model of Apo-enzyme (without 102 carmofur) was calculated using MODELLER version 9.10 [25]. Before docking, crystallographic water molecules and bound ligands were removed from 3D structures. The protonation states of acidic and basic residues were determined under $\mathrm{pH} 7.0$ conditions and analyzed by the $\mathrm{H}++$ server [17]. Next, the Modell structure was subjected to Discovery studio for optimization and minimization. Finally, validate the modeled structure using the UCLA-DOE server (http://servicesn.mbi.ucla.edu/) [18 19 20]. The modeled 3D structure was then validated and confirmed by using the RAMPAGE, ERRAT, and Verify3D online servers. After validating the homology models, we resolved the issue of mismatched residue and missed structure across the two models. The SMILES of the three natural polyphenols diferuloylmethane, demethoxycurcumin, and bisdemethoxycurcumin were obtained from Pub Chem [21] and their accession numbers are DB969516, DB5469424, and DB5315472 respectively. These SMILES were converted to PDB format with 3 D coordinates using Open Babel [22], an open-source chemical toolbox for the inter conversion of chemical structures.

116 The experiments were performed with Auto-Dock 4.2 according to the previous study procedure 117 [23]. The work was separated into two parts; the first was prepared ligand and protein pdbqt file and Docking Parameter file using Auto-Dock 4.2. The second was performed molecular docking and finally, the results were analyzed. Fourteen refined 3D polyphenols structures were screened for binding affinity and selectivity toward protein. To cover the whole protein structure global docking was conducted with the spacing of $0.5 \AA$. Subsequently, rigid or flexible docking of the target was performed and then the complexes with the lowest binding energies are selected. The genetic algorithm was used to evaluate parameters with the default setting, and Lamarckian GA 
125 genetic algorithm runs using the Lamarckian genetic algorithm conformational search, with the 126 population size of 300, 2500000 maximum numbers of energy evaluations, and 27000 127 generations per run. The best protein-polyphenols complexes were selected according to the 128 molecular docking results including binding energy, root mean square deviation, and type of 129 favorable interactions and binding sites.

130 Set Simulation Parameters and Molecular Dynamics

131 To explore the conformational flexibility of best hit compounds against free protein, an MD 132 simulation study was employed using NAMD 2.3 tool on window operating system [24]. Each 133 complex was separated for generating topology and coordinates. The protein topology and 134 coordinate files were lacking in the Amber force field prepared by the general amber force field 135 (CgenFF) Server [25], while the ligands topology and coordinate files were generated using 136 Open Babel [22]. The generated NAMD compatible files for the proteins and the ligands were 137 then merged, and then complex subjected to solvated, minimized, and equilibrated. The systems 138 were solvated in a cubic water-box with the explicit solvation model TIP3P.20 we used a 139 distance of $1.0 \AA$ between the cell wall and the solvated atoms of the system. Counter-ions were 140 also added to neutralize the system. The energy minimization (n steps $=5000$ ) was conducted 141 using the steepest descent approach (1000ps) for each protein-ligand complex. Particle Mesh 142 Ewald (PME) method was employed for energy calculation and for electrostatic and Van der 143 Waals interactions; cut-off distance for the short-range Van der Waals was set to $10 \AA$, where 144 Coulomb cut-off and neighbor list were fixed at $8 \AA$. Finally, a 50ns molecular dynamics 145 simulation was carried out for all the complexes with n steps 1000000. The RMSD, hydrogen 146 bond distribution, and RMSF analysis were carried out using MS Excel (2016), VMD, and 147 UCSF Chimera 1.10.1 software. Trajectory snapshots were stored at every $0.2 \mathrm{ps}$ during the 148 simulation period, and 3D coordinate files were harvested after every $2 \mathrm{fs}$ for post-dynamic 149 analysis.

150 MM-PBSA Approach Interaction energy

151 The binding energy of Mpro-Curcumin derivative polyphenols complexes was calculated using 152 the molecular mechanics Poisson-Boltzmann surface area (MM-PBSA) method [26]. Free 153 energy of solvation (TP3 solvent model) and molecular mechanics potential energy was 154 calculated. In this study, snapshots were taken from MD simulation used for calculating binding 155 free energy. The individual contributions of protein residues to the three energetic components 
156 were determined through per-residue decomposition. The temperature used for Poisson157 Boltzmann calculation was set to $300 \mathrm{~K}$.

158 Prediction of ADME by Computational analysis

159 ADME analysis of Bisdemethoxycurcumin, Demethoxycurcumin, and Diferuloylmethane at $\mathrm{pH}$ 1607 was examined using online software tools [27]. The important parameters related to ADME 161 properties such as Proudfoot's rule of five, pharmacokinetic properties, drug likeliness, molar 162 refractivity, and solubility of the drug was deliberated [28].

163

164

165

166

167

168

169

170

171

172

173

174

175

176

177

178

179

180

181

Peer] reviewing PDF | (2020:05:48854:4:0:CHECK 15 May 2021) 


\section{Discussion}

183 Until now, no specific antiviral medication was discovered for COVID-19 the only control 184 measure includes proper hand washing, social distancing, mouth and nose masking, travel

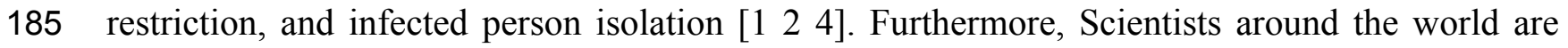
186 developing many potential vaccines for COVID-19 and the biggest vaccination campaign in 187 history is underway. However, according to the world meter report, the coronavirus COVID-19 188 is still affecting 219 countries and worldwide 160686749 peoples infected and 3335948 people 189 died until May 14, 2021, 20:04 GMT. In the context of Ethiopia, this virus died about 3,951 190 people and infected 264,960 people. Viruses continually change through mutations and the gene 191 encoding the S protein of SARS-CoV-2, various mutations have been reported and recently, the 192 United Kingdom (UK) (VOC-202012/01 or VUI-202012/01 or B.1.1.7), Indian (B.1.617) and 193 South Africa (501Y.V2 or 20C/501Y.V2B.1.351) have faced a rapid increase in COVID-19 194 mediated by new variants [48 49]. One way of recovering from this problem could be to identify 195 potent COVID-19 inhibitors is urgently needed, as the situation getting worst. In this regard, 196 plants serve as the source of therapeutic ingredients in the history of the human race. As far as 197 we know, little previous research has investigated curcumin sp polyphenols inhibitory effect on 198 COVID-19. Curcumin consists of three major compounds, namely, diferuloylmethane (60-70\%), 199 demethoxycurcumin (20-27\%), and Bisdemethoxycurcumin (10-15\%) [29 30]. The review 200 showed curcumin derived polyphenols exhibit antiviral activity for influenza, hepatitis $\mathrm{C}$ virus, 201 HIV virus, anti-inflammatory, antibacterial, and antioxidant properties and also exert substantial 202 anti carcinogenic activities [ $\left[\begin{array}{lll}29 & 30 & 31\end{array}\right]$. Wet lab experimental approaches for the study of 203 interactions between therapeutic substance and target proteins are not cheap, not safe, labors, and 204 time-consuming [32 33]. There are many alternative methods are available for solving these 205 problems. In this study, we proposed the combinational strategy of docking and molecular 206 dynamics simulation. A series of recent studies have indicated that combinational strategy of the 207 docking and molecular dynamic simulations techniques identify the targets for several bioactive 208 compounds whose in vivo targets are unknown including COVID-19 [38 4041 ]. The aim of this 209 study is to investigate the binding affinity of three Curcumin derived polyphenols against 210 COVID-19 the main protease (Mpro) binding pocket (Mpro) and the identification of important 211 residues for interaction with curcumin derived polyphenols. For this purpose, we used protein212 carmofur (PDB code: 7BUY) as a model to offer evidence that could elucidate the mechanisms 
213 of curcumin sp polyphenols inhibition. At the beginning of this study, curcumin sp-derived 214 polyphenols were subjected to ADMETox evaluation. According to Proudfooti's rule states that 215 for any compound to be selected as a potential drug it should have; molar refractivity between 40 216 and 130, Less than 10 hydrogen bond acceptors, less than 5 hydrogen bond donors, high 217 lipophilicity (expressed as $\operatorname{LogP} \leq 5$ ) and Molecular mass $<500$ Dalton. Therefore, three selected 218 curcumin sp-derived polyphenols used in this study passed all the five criteria mentioned in 219 Proudfoot's rule (Table 3 supp.). Demethoxycurcumin and bisdemethoxycurcumin polyphenol 220 passed the ADME evaluation and throughout the screening, only Diferuloylmethane passed the 221 predicted toxicity evaluation. Thus this study suggests that these phytochemicals have the 222 potential to work effectively as drugs. Next, to validate our docking methodology crystal 223 structure of COVID-19 main protease binding pocket (Mpro) (PDB code: 7BUY) with carmofur 224 bound at the binding pocket was retrieved from PDB and re-dock with carmofur. The values of 225 the RMSD between the Mpro crystals structure (RMSD 1.6) and the re-dock structures (RMSD 226 1.9) confirmed close-match between docked and original structure. This confirmed our model 227 structure is good quality and continued docking our work. Molecular docking studies showed 228 that curcumin polyphenols have binding energy values between -12.17 to $-12.32 \mathrm{kcalmol}^{-1}$ as shown in table 1 (Table 1). Recent theoretical developments have revealed that Docking studies on different traditional medicine inhibitors activity for the SARS-CoV-2 protease. Several studies have been reported the binding energy of molecules less than or/and higher values compared to this study. Cherrak et al. reported in the literature that Quercetin-3-O-rhamnoside showed the highest binding affinity $-7 \mathrm{kcal} / \mathrm{mol}^{-1}$ [34]. Moreover, it was observed that curcumin exhibited the highest binding free energy of $-18.21 \mathrm{kcal} / \mathrm{mol}^{-1}$ in different COVID-19 main protease, which is targeted in this study [35]. And also, docking of SARS-CoV-2 spike protein (PDB ID: 6LU7) with nelfinavir, lopinavir, kaempferol, quercetin,luteolin-7237 glucoside,demethoxycurcumin, naringenin, apigenin-7-glucoside, oleuropein, curcumin, catechin, epicatechin-gallate, gingerol, gingerol, and allicin binding energies ranges between -4.03 to -7.6 $\mathrm{kcal} / \mathrm{mol}^{-1}$ [36]. Recently, Computational docking of 14 compounds representing flavonoids, phenolic acids and terpenes from honey and propolis with two different targets from COVID-19

241 showed binding affinity ranges between -5.6 to $-7.8 \mathrm{kcal} / \mathrm{mol}^{-1}$ [48]. Bisdemethoxycurcumin, 242 Demethoxycurcumin and Diferuloylmethane revealed a minimum of two hydrogen bonds and a 
244 line with previous studies, hydrogen bonds are the most important bonds in determining the 245 binding affinity, selectivity, and stabilization effect of curcumin derived polyphenols with Mpro 246 [37]. The inhibition constant (Ki) is higher for Demethoxycurcumin (281.22uM) than 247 Bisdemethoxycurcumin $(98.83 \mathrm{uM})$ and Diferuloylmethane $(156.42 \mathrm{uM})$ as shown in table 1. 248 Prior research suggests that the smaller the $\mathrm{Ki}$, the greater the binding affinity and the smaller 249 amount of medication needed to inhibit the activity of that enzyme [33]. The RMSD values 250 obtained for the lowest-energy poses predicted for each polyphenol are shown in Table 1 and 251 average RMSD results ranging from 57.0 to $70.0 \AA$ RMSD. But this is not relevant data for this 252 study.

253 Furthermore, residues forming stable contact with polyphenols include Phe294, Gln110, Gln240, 254 Gln192 Asn142, Lys137, Thr199, and Leu272 with probabilities larger than 85\% (Figure 1 table 255 1). Bisdemethoxycurcumin and Demethoxycurcumin have lost hydrophobic interaction with 256 Pro293 with Tyr239, respectively (figure 1). Besides this, a total of eight residue pairs that have

257 258 259 260 261 262 263 264 265 266 267 268 269 270 271 272 274 273 Val202, Gly109, Pro293, Ile249, Pro252, Thr292, Ile200, and Cys145 their contact probabilities average distances smaller than $10.0 \AA$ within free enzyme residues and Mpro-polyphenols complexes binding pocket residues and surface residues reported (Table 2). This implies that residue from both surface and binding pocket protein is involved in direct contact with curcumin derived polyphenols. Furthermore, the inter-residue distances between the binding pocket and surface residues in Mpro-bisdemethoxycurcumin and Mpro-demethoxycurcumin complex are slightly different from that of Mpro-diferuloylmethane, particularly for Pro252/Lys137, Thr292/Thr199, Ile200/Leu272, and Arg188/Phe294 residues (Table 2). In line with previous studies, residue serine interacts with residue Phe140 and Glu166 to stabilize the enzymepolyphenols complex binding site [7]. For residues Gln192, Leu167, Ser144, Gly143, His114, Pro168, His41, and Cys145, their contact probabilities with bisdemethoxycurcumin were increased significantly and Cys145 is a key catalytic residue which is consistent with a previous study [7]. Residues forming stable contact with polyphenol include Phe294, Gln110, Gln240, Gln192 Asn142 Lys137 Thr199, and Leu272 the complexes with probabilities larger than 85\% (Figure 1). Also, for residues Glu288, Asp289, Leu287 Arg131, Tyr239, Leu271, Asn274, 71 Gly275, and Leu286 the probabilities of contacts between demethoxycurcumin increased 72 significantly after binding and they also form stable contacts (figure 1). For residues Arg188, 274 with bisdemethoxycurcumin were increased significantly and Cys145 is a key catalytic residue 
275 which is consistent with a previous study [7]. Pro168, His41 and Val116 residues contact 276 probabilities between demethoxycurcumin-Mpro and bisdemethoxycurcumin-Mpro decreased 277 (Table 3). Previous studies have shown COVID-19 main protease possesses a dynamic binding 278 pocket loop comprising domain-I (residues 10 to 99) and domain-II (residues 100 to 184), in 279 which most of the residues in this study laid [7 42 43].

280 To examine the stability and conformational dynamics of the Mpro-three curcumin derived 281 polyphenols complex and free Mpro, we calculated the RMSD for the backbones of all residues 282 (Figure 1; Figure 2). In this study, much attention has been given to the impact of curcumin 283 derived polyphenols diferuloylmethane, demethoxycurcumin, and bisdemethoxycurcumin on the 284 structure and dynamics of the main protease (Mpro). Here free Mpro has a significantly large 285 root-mean-square deviation compare to curcumin sp polyphenols binding the Mpro pocket 286 domain. The time evolution plot of RMSD displays the complex structure of diferuloylmethane287 Mpro, demethoxycurcumin-Mpro, and bisdemethoxycurcumin-Mpro attain equilibrium at 288 1000ps and remains stable up to 5000ps (Figure 1). In particular, the complex structure with 289 demethoxycurcumin and bisdemethoxycurcumin is largely stabilized around 3000ps to 6000ps 290 (Figure 2). Likewise, its fluctuation main protease complex is smaller, especially in the segment 291 Asn226 to Gly252, which is the active-site loop region which is consistent with the previous 292 study discussed (Figure 3). Particularly, binding of the bisdemethoxycurcumin was observed to 293 decrease RMSF of some segments (30 to 100, 200 to 250, and 250 to 300) and increase that of 294 the segment (100 to 150) (Figure 3). The higher value of RMSF depicted that the structure has 295 some flexible regions while the lower value of RMSF indicated that the structure was good in 296 terms of secondary structure [22]. Therefore, the result revealed the interactive stabilizing effect 297 of Demethoxycurcumin and Bisdemethoxycurcumin. Also, the impact of these polyphenols on 298 the structure and dynamics of the Main protease binding pocket domain region was further 299 regulated by a hydrogen bond. Here, we also examine the time evolution of hydrogen bond $(\mathrm{H}-$ 300 bond) formed between the Mpro and curcumin sp derived polyphenols (Figure 4). We found that 301 the trajectory of bisdemethoxycurcumin revealed five H-bonds with Mpro, three H-bonds were 302 seen consistently during the simulation (Figure 4). Demethoxycurcumin revealed the presence of 303 ten H-bonds with Mpro but, three H-bond can be seen retained throughout the simulation, the H304 bond plot of diferuloylmethane shows a maximum of seven H-bond interactions with Mpro, but 305 only two seen retained throughout the simulation (Figure 4), while the carmofur showed the 
306 lesser number of hydrogen bonds [7]. The strong hydrogen bonding interactions between the

307 demethoxycurcumin-Mpro and bisdemethoxycurcumin-Mpro may be a potential inhibitor [21].

308 This study may contribute to the understanding of the structure-based design of COVID-19

309 therapy with curcumin derived polyphenols. Future investigations are necessary to validate the

310 kinds of conclusions that can be drawn from this study.

\section{Conclusions}

312 Until now, Great progress has been made in Computational methods involving drug target

313 identification in a paradigm change in both industry and academics. Computational techniques,

314 for example, data mining, homology modeling, MD simulation, cheminformatics, docking, and

315 QSAR modeling have provided powerful techniques for target identification, drug discovery, and

316 optimization. First, curcumin derived polyphenols were subjected to in silico ADMETox and we

317 found the potential ability to work effectively as drugs. Based on the results of the present study,

318 it can be concluded that the investigated curcumin derived polyphenols could interfere with the 319 important residues in the enzymatic binding pocket to inhibit the main protease enzyme COVID32019 virus. Demethoxycurcumin and bisdemethoxycurcumin polyphenols are identified to have 321 inhibitory activities against novel COVID-19 main protease. Bisdemethoxycurcumin has a 322 stronger bond and high affinity with the Main protease (Mpro). Furthermore, the average RMSD 323 values of the backbone atoms in docked curcumin sp derived polyphenols were calculated from 324 10000ps and showed stable RMSD values between $1 \mathrm{~nm}$ to $2 \mathrm{~nm}$ for Bisdemethoxycurcumin and 325 Demethoxycurcumin at the reasonably consistent temperature ( 300 K) and pressure (1bar), 326 whereas diferuloylmethane complex showed RMSD value between 5.5 and 6.2 with same cut-off 327 parameters. These data validated that the docked Mpro-Bisdemethoxycurcumin complexes and 328 Mpro-Demethoxycurcumin complexes are more stable than the Mpro-diferuloylmethane 329 complex. To completely investigate the effects of curcumin derived polyphenols, this study 330 recommended the roles of polyphenols identified for further exploration in a wet lab experiment.

\section{Acknowledgement}

332 For the success of this work we would like to acknowledge effort of our family for their 333 inspiration. We acknowledge the university of Addis Ababa Science and Technology for using 334 computational resources. 


\section{References}

337 1. World Health Organization. (2020). Coronavirus Disease 2019 (COVID-19) Situation Report 338 -43. 2020. Available online: https://www.who.int/emergencies/diseases/novel-coronavirus339 2019/situation-reports

340 2. Guarner J. (2020). Three emerging coronaviruses in two decades: the story of SARS, MERS, 341 and now COVID-19. American Journal of Clinical Pathology; 153: 420-421.

342 3. Chen Y, Liu Q and Guo D. (2020). Emerging coronaviruses genome structure, replication and 343 pathogenesis. J Med Virol; 92(10):2249.

344 4. Zhao Q, Weber E \& Yang H. (2013). Recent developments on coronavirus main protease/3C 345 like protease inhibitors. Recent Patents on anti-Infective Drug Discovery; 8(2): 150-156.

346 5. Pal M, Berhanu G, Desalegn C and Kandi V. (2020). Severe Acute Respiratory Syndrome 347 Coronavirus-2 (SARS-CoV-2): An Update. Cureus; 12(3): e7423.

348 6. Woo PCY, Huang Y, Lau SKP and Yuen KY. (2010). Coronavirus Genomics and 349 Bioinformatics Analysis. Viruses; 2(8):1804-20.

350 7. Jin Z, Zhao Y, Sun Y, Zhang B, Wang H, Wu Y, Zhu Y, Zhu C, Hu T, Du X, Duan Y et al. 351 (2020). Structural basis for the inhibition of SARS-CoV-2 main protease by antineoplastic drug 352 Carmofur. Nature structural and molecular biology; 27: 529-532

353 8. Muralidharan N, Sakthivel R, Velmurugan D \& Gromiha MM. (2020). Computational studies 354 of drug repurposing and synergism of lopinavir, oseltamivir and ritonavir binding with SARS355 CoV-2 protease against COVID-19. Journal of Biomolecular Structure and Dynamics; 16; 1-6.

356 9. Liu X, Zhang B, Jin Z, Yang H, Rao Z. (2020). The Crystal Structure of COVID-19 Main 357 Protease in Complex with an Inhibitor N3. J. Chem. Inf. Model; 60 (7): 3593-3602

358 10. WHO Director-General's opening remarks at the media briefing on COVID-19 - 3 March 3592020 - World Health Organization, March 3, 2020

360 11. Islam MT, Khan MR \& Mishra SK. (2019). An updated literature-based review: 361 Phytochemistry, pharmacology and therapeutic promises of Nigella sativa L. Oriental Pharmacy 362 and Experimental Medicine; 19(2): 115-115.

363 12. Forni C, Facchiano F, Bartoli M, Pieretti S, Facchiano A, D'Arcangelo D, Norelli S, Valle 364 G, Nisini R, Beninati S, Tabolacci C \& Jadeja RN. (2019). Beneficial Role of Phytochemicals on 
365 Oxidative Stress and Age-Related Diseases. BioMed Research International: 2019(4, article 366 918).

367 13. Hatcher H, Planalp R, Cho J, Torti FM and Torti SV. Curcumin: from ancient medicine to 368 current clinical trials. Cell Mol Life Science; 65(11):1631-52.

369 14. Anbarasu K and Jayanthi S. (2018). Identification of curcumin derivatives as human LMTK3 370 inhibitors for breast cancer: a docking, dynamics, and MM/PBSA approach, Springer; 8(5):228371239.

372 15. Mounce BC, Cesaro T, Carrau L, Vallet T \& Vignuzzi M. (2017). Curcumin inhibits Zika 373 and chikungunya virus infection by inhibiting cell binding. Antiviral Research; 142: 148-157.

374 16. Praditya D, Kirchhoff L, Bruning J, Rachmawati H, Steinmann J \& Steinmann E. (2019).

375 Anti-infective properties of the golden spice curcumin. Frontiers in Microbiology; 10: 912.

376 17. Anandakrishnan R, Aguilar B and V. Onufriev A. (2012). H++ 3.0: automating pK 377 prediction and the preparation of biomolecular structures for atomistic molecular modeling and 378 simulation. Nucleic Acids Res; 4: 537-541.

379 18. Benkert P, Michael K, Torsten S. (2009). QMEAN server for protein model quality 380 estimation. Nucleic Acids Res; 37:510-514

381 19. Colovos $\mathrm{C}$ and Yeates TO. (1993).Verification of protein structures: patterns of nonbonded 382 atomic interactions. Protein Science; 2(9):1511-9.

383 20. Pontius J, Richelle J and Wodak SJ. (1996). Deviations from standard atomic volumes as a 384 quality measure for protein crystal structures. $J$ Mol Biol; 264(1):121-36.

385 21. Bolton EE, Wang Y, Thiessen PA and Bryant SH. (2008). PubChem: integrated platform of 386 small molecules and biological activities. Annual Reports in Computational Chemistry. Elsevier; 387 4(2008): 217-241.

388 22. O'Boyle, NM, Banck M, James CA, Morley C, Vandermeersch T \& Hutchison GR. (2011). 389 Open Babel: An open chemical toolbox. Journal of Cheminformatics; 3(1):33.

390 23. Morris GM, Huey R, Lindstrom W, Sanner MF, Belew RK, Goodsell DS, Olson AJ. (2009). 391 AutoDock4 and AutoDockTools4: Automated docking with selective receptor flexibility. $J$ 392 Comput Chem; 30(16):2785-91.

393 24. Nelson M, Humphrey W, Gursoy A, Dalke A, V.Kalé L, D. Skeel R and Schulten K. (1996). 394 NAMD - A parallel, object-oriented molecular dynamics program. International Journal of 395 Supercomputer Applications and High Performance Computing; 10:251-268. 
396 397 398 399 400

401 402 403 404 405 406

407

408 409

410

411

412

413

414

415

416

417

418

419

420

421

422

423

424

425

25. Vanommeslaeghe K, Raman EP and MacKerell AD. (2012). Automation of the CHARMM General Force Field (CGenFF) II: assignment of bonded parameters and partial atomic charges. Journal of chemical information and modeling; 52 (12): 3155-3168.

26. Kumari R, Kumari R \& Lynn A. (2014). g_mmpbsa--a GROMACS tool for high throughput MM-PBSA calculations. J Chem Inf Model; 54(7):1951-1962.

27. B. Baell J and A. Holloway G. (2012). New Substructure Filters for Removal of Pan Assay Interference Compounds (PAINS) from Screening Libraries and for Their Exclusion in Bioassays. J. Med. Chem; 53 (7): 2719-2740.

28. Daina A, Michielin O \& Zoete V. (2017). SwissADME: a free web tool to evaluate pharmacokinetics, drug-likeness and medicinal chemistry friendliness of small molecules. Sci. Rep; 7:42717.

29. Heger M, van Golen RF, Broekgaarden M and Michel MC. (2013). The molecular basis for the pharmacokinetics and pharmacodynamics of curcumin and its metabolites in relation to cancer. Pharmacological Reviews; 66(1):222-307

30. Nelson KM, Dahlin JL, Bisson J, Graham J, Pauli GF and Walters MA. (2017). The essential medicinal chemistry of curcumin. J Med Chem; 60(5):1620-1637.

31. Praditya D, Kirchhoff L, Brüning J, Rachmawati H, Steinmannand J and Steinmann E. (2019). Anti-infective Properties of the Golden Spice Curcumin. Front Microbiol; 10: 912.

32. SARBASHRI BANK. (2020). In-silico analysis of potential interaction of drugs and the SARS-CoV-2 spike protein. Research square. DOI: https://doi.org/10.21203/rs.3.rs-30401/v1

33. Bachmann KA and Lewis JD. (2005). Predicting inhibitory drug-drug interactions and evaluating drug interaction reports using inhibition constants. Ann Pharmacother; 39:1064-72.

34. Cherrak SA, Merzouk H and Mokhtari-Soulimane N. (2020). Potential bioactive glycosylated flavonoids as SARS-CoV-2 main protease inhibitors: A molecular docking and simulation studies. Plos One; 15 (10), e0240653. doi:10.1371/journal.pone.0240653

35. Adeoye A. (2020). Pharmacoinformatics and hypothetical studies on allicin, curcumin, and gingerol as potential candidates against COVID-19-associated proteases. Journal of biomolecular Structure \& Dynamics. DOI: 10.1080/07391102.2020.1813630

36. Khaerunnisa S, Kurniawan H, Awaluddin R, Suhartati S and Soetjipto S. (2020). Potential Inhibitor of COVID-19 Main Protease (Mpro) from Several Medicinal Plant Compounds by 
426 Molecular Docking Study. Hindawi; $\quad$ Volume 2020 |Article $\quad$ ID 6307457

427 https://doi.org/10.1155/2020/6307457

428 37. Baby K, Maity S, Mehta CH, Suresh A, Nayak UY and Nayak Y. (2020). Targeting SARS-

429 CoV-2 main protease: a computational drug repurposing study. Archives Medical Res. 52 (1), 430 38-47. doi:10.1016/j.arcmed.2020.09.013

431 38. Rahman MS, Hoque MN, Islam MR, Akter S, Rubayet U, Alam ASM, Siddique MA, Saha 432 O, Rahaman MM, Sultana M, Crandall KA and Hossain MA. (2020). Epitope-based chimeric 433 peptide vaccine design against $\mathrm{S}, \mathrm{M}$ and $\mathrm{E}$ proteins of SARS-CoV-2, the etiologic agent of 434 COVID-19 pandemic: an in silico approach. PeerJ 8:e9572 https://doi.org/10.7717/peerj.9572

435 39. Hoque MN, Chaudhury A, Akanda MAM, Hossain MA and Islam MT. (2020). Genomic 436 diversity and evolution, diagnosis, prevention, and therapeutics of the pandemic COVID-19 437 disease. PeerJ 8:e9689 https://doi.org/10.7717/peerj.9689

438 40. Fiesco-Sepúlveda KY and Serrano-Bermúdez LM. (2020). Contributions of Latin American 439 researchers in the understanding of the novel coronavirus outbreak: a literature review. PeerJ; 440 8:e9332 https://doi.org/10.7717/peerj.9332

441 41. Cortés-García CJ, Chacón-García L, Mejía-Benavides JE and Díaz-Cervantes E. (2020). 442 Tackling the SARS-CoV-2 main protease using hybrid derivatives of 1, 5-disubstituted tetrazole443 1, 2, 3-triazoles: an in silico assay. PeerJ; Physical Chemistry 2:e10 444 https://doi.org/10.7717/peerj-pchem.10

445 42. Kouznetsova VL, Zhang A, Tatineni M, Miller MA and Tsigelny IF. (2020). Potential 446 COVID-19 papain-like protease PLpro inhibitors: repurposing FDA-approved drugs. PeerJ; 447 8:e9965 https://doi.org/10.7717/peerj.9965

448 43. Kandeel M, Kitade $\mathrm{Y}$ and Almubarak A. (2020). Repurposing FDA-approved 449 phytomedicines, natural products, antivirals and cell protectives against SARS-CoV-2 RNA450 dependent RNA-polymerase. PeerJ; 8:e10480 https://doi.org/10.7717/peerj.10480

451 44. Elaswad A, Fawzy M, Basiouni S and Shehata A. (2020). Mutational spectra of SARS-CoV4522 isolated from animals. PeerJ; 8:e10609 https://doi.org/10.7717/peerj.10609

453 45. Parlikar A, Kalia K, Sinha S, Patnaik S, Sharma N, Vemuri SG and Sharma G. (2020). 454 Understanding genomic diversity, pan-genome, and evolution of SARS-CoV-2. PeerJ; 8:e9576 455 https://doi.org/10.7717/peerj.9576 
456 46. Bin Emran T, Rahman MA, Nasir Uddin MM, Dash R, Firoz Hossen M, Mohiuddin M and 457 Rashadul Alam m. (2015). Molecular docking and inhibition studies on the interactions of 458 Bacopa monnieri's potent phytochemicals against pathogenic Staphylococcus aureus. DARU 459 Journal of Pharmaceutical Sciences; 23(1): 1-8

460 47. Dash R, Bin Emran T, Nasir Uddin MM, Islam A and Junaid M. (2014). Molecular docking 461 of fisetin with AD associated AChE, ABAD and BACE1 proteins. Bioinformation; 10(9): 562

462 48. Dawood AA. (2020). Mutated COVID-19 may foretell a great risk for mankind in the future. 463 New Microbes New Infect; 35:100673.

464 49. Korber B, Fischer WM, Gnanakaran S, Yoon H, Theiler J, Abfalterer W, Hengartner N, 465 Giorgi EE, Bhattacharya T, Foley B et al. (2020). Tracking Changes in SARS-CoV-2 Spike: 466 Evidence that D614G Increases Infectivity of the COVID-19 Virus. Cell; 182:812-827.e19. 
Figure 1

Figure 1. Plots of Root-mean-square deviations of free SARS CoV-2 main protease (Mpro) (Blue) and the complex of SARS CoV-2 main protease (7BUY) (Red) with three polyphenols along the MD simulation time
(A) free SARS CoV-2 main protease (Mpro) (Blue)
(B) the complex of SARS CoV-2 main protease (7BUY) (Red) with three polyphenols 


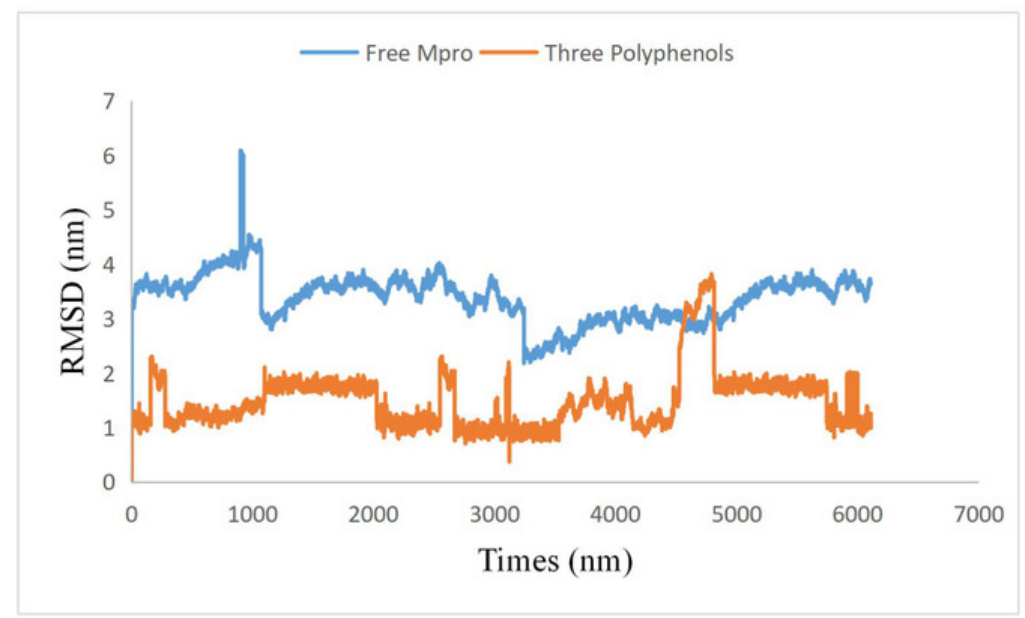


Figure 2

Figure 2. Plots of Root-mean-square deviations of free main CoV-2 protease (Mpro) (Gray) and the complex of Mpro with Bisdemethoxycurcumin (Yellow), Demethoxycurcumin (Blue) and Diferuloylmethane (Red) along the MD simulation time for three individual pol

(A) free main CoV-2 protease (Mpro) (Gray) and the complex of Mpro with

Bisdemethoxycurcumin (Yellow), (B) Demethoxycurcumin (Blue) and (C) Diferuloylmethane (Red) 


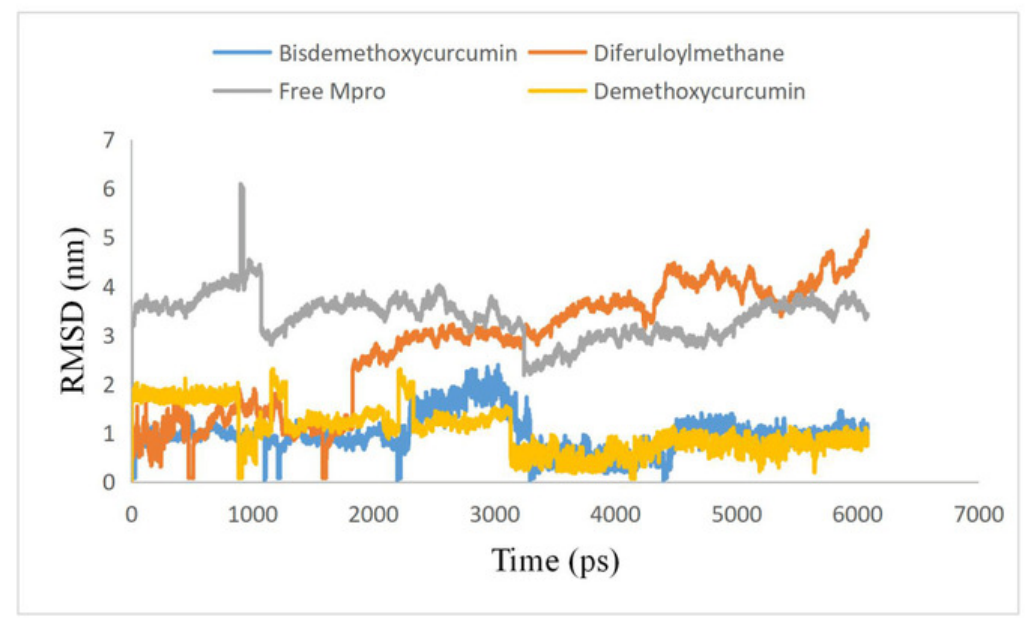




\section{Figure 3}

Figure 3. RMSF plot of free main CoV-2 protease (Mpro)(Yellow) and the complex of Mpro with Bisdemethoxycurcumin (Red), Demethoxycurcumin (Blue) and Diferuloylmethane (Gray) along the MD simulation time for three individual polyphenols.

(A) free main CoV-2 protease (Mpro)(Yellow) and (B) the complex of Mpro with Bisdemethoxycurcumin (Red), (C) Demethoxycurcumin (Blue) and (D) Diferuloylmethane (Gray) 


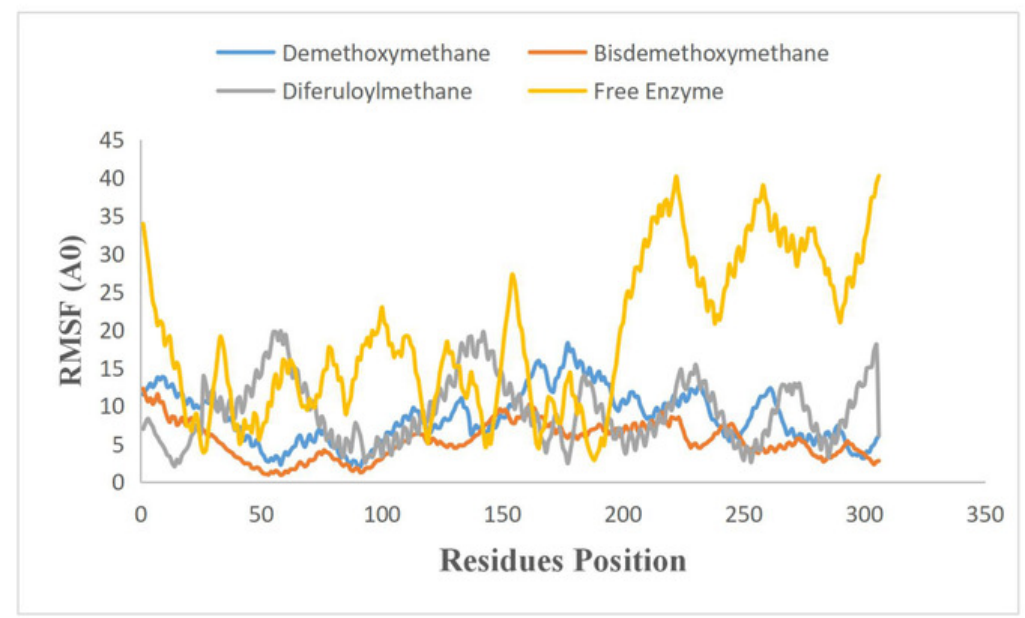




\section{Figure 4}

Figure 4. Number of hydrogen bond present in Bisdemethoxycurcumin-SARS-CoV-2 main protease (Yellow), Demethoxycurcumin-SARS-CoV-2 main protease (Gray), free SARS-CoV-2 main protease (Red) and diferuloylmethane-main protease (Blue).

(A) Bisdemethoxycurcumin-SARS-CoV-2 main protease (Yellow), (B) DemethoxycurcuminSARS-CoV-2 main protease (Gray), (C) free SARS-CoV-2 main protease (Red) and (D) diferuloylmethane-main protease (Blue). 


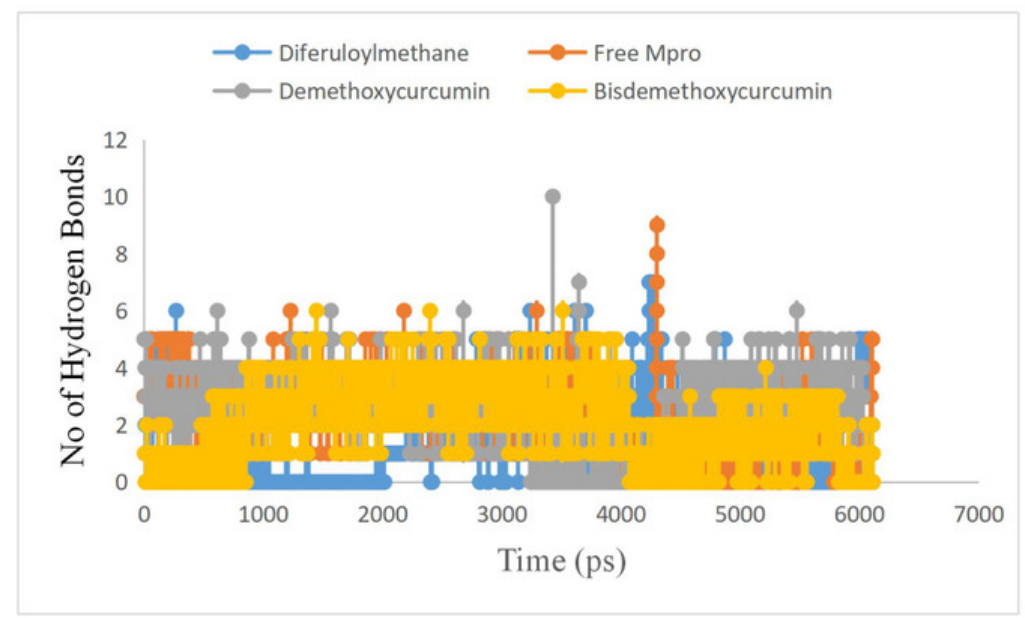




\section{Table $\mathbf{1}$ (on next page)}

Table 1 shows molecular docking results to determine the SARS CoV-2 main protease (Mpro) binding pocket affinity towards selected curcumin sp derived polyphenols at $298.15 \mathrm{~K}$ 
1

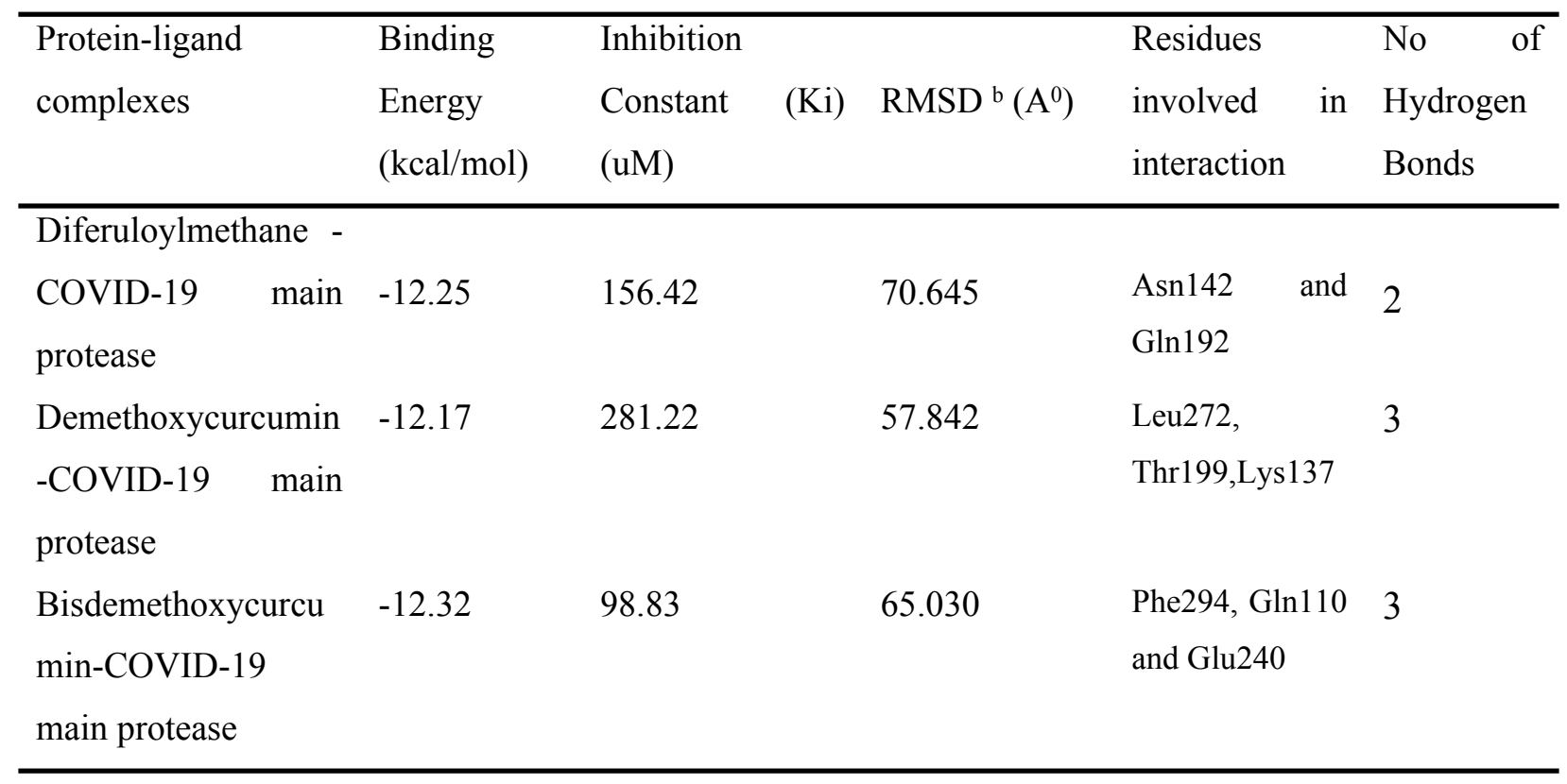

2 


\section{Table 2 (on next page)}

Table 2 Inter-residue distances and corresponding standard deviations. In each residue pair, one belongs to the binding pocket loop region and the other belongs to surface part of the enzyme 
1

\begin{tabular}{cccc}
\hline Residues & Free enzyme & $\begin{array}{c}\text { Enzyme- } \\
\text { Demethoxycurcumin } \\
\text { complex }\end{array}$ & $\begin{array}{c}\text { Enzyme- } \\
\text { Bisdemethoxycurcumin } \\
\text { complex }\end{array}$ \\
\hline Arg188- Phe294 & $5.154 \pm 1.531$ & $5.937 \pm 1.604$ & $4.5 \pm 1.10$ \\
Val202- Gln110 & $9.419 \pm 1.440$ & $8.455 \pm 1.090$ & $9.25 \pm 1.440$ \\
Gly109- Gln240 & $10.111 \pm 1.838$ & $8.296 \pm 1.459$ & $11.31 \pm 1.14$ \\
Pro293- Gln192 & $8.570 \pm 1.663$ & $7.095 \pm 1.022$ & $5.13 \pm 1.04$ \\
Ile249- Asn142 & $9.170 \pm 5.015$ & $10.485 \pm 1.560$ & $6.71 \pm 0.44$ \\
Pro252- Lys137 & $7.11 \pm 0.02$ & $8.5 \pm 1.4$ & $5.63 \pm 1.04$ \\
Thr292- Thr199 & $5.109 \pm 0.440$ & $6.039 \pm 0.940$ & $4.110 \pm 0.230$ \\
Ile200- Leu272 & $7.213 \pm 0.018$ & $9.216 \pm 0.218$ & $6.121 \pm 0.618$ \\
\hline
\end{tabular}


Table 3(on next page)

Table 3 residues forming contacts with diferuloylmethane polyphenol, demethoxycurcumin polyphenol and bisdemethoxycurcumin polyphenol in binary complexes. 


\begin{tabular}{cccc}
\hline Residues & $\begin{array}{c}\text { Enzyme- } \\
\text { Demethoxycurcu } \\
\text { min complex }\end{array}$ & $\begin{array}{c}\text { Enzyme } \\
\text { Bisdemethoxycurcumi } \\
\text { n complex }\end{array}$ & $\begin{array}{c}\text { Enzyme- } \\
\text { diferuloylmethane } \\
\text { complex }\end{array}$ \\
\hline Gln192 & $0.70 \pm 0.01$ & $0.85 \pm 0.01$ & $0.45 \pm 0.02$ \\
Leu167 & $0.90 \pm 0.03$ & $0.98 \pm 0.004$ & $0.60 \pm 0.05$ \\
Tyr239 & $0.60 \pm 0 . .07$ & $0.80 \pm 0.01$ & $0.30 \pm 0.01$ \\
Asp289 & $0.70 \pm 0.19$ & $0.90 \pm 0.004$ & $0.60 \pm 0.1$ \\
Arg188 & $0.75 \pm 0.011$ & $0.80 \pm 0.05$ & $0.73 \pm 0.09$ \\
Glu166 & $0.80 \pm 0.04$ & $0.80 \pm 0.01$ & $0.90 \pm 0.01$ \\
Glu288 & $1.00 \pm 0.06$ & $0.50 \pm 0.01$ & $0.60 \pm 0.12$ \\
His114 & $1.00 \pm 0.00$ & $1.00 \pm 0.00$ & $0.50 \pm 0.03$ \\
Gln189 & $0.70 \pm 0.05$ & $0.90 \pm 0.00$ & $1.00 \pm 0.09$ \\
Pro168 & $0.30 \pm 0.01$ & $1.00 \pm 0.00$ & $0.85 \pm 0.05$ \\
Gln189 & $0.45 \pm 0.02$ & $1.00 \pm 0.00$ & $0.40 \pm 0.16$ \\
Cys145 & $0.75 \pm 0.09$ & $0.90 \pm 0.01$ & $0.60 \pm 0.06$ \\
Met165 & $0.80 \pm 0.1$ & $0.85 \pm 0.12$ & $0.50 . \pm 0.12$ \\
His 164 & $0.40 \pm 0.00$ & $0.70 \pm 0.01$ & $1.00 \pm 0.04$ \\
Asp187 & $0.90 \pm 0.03$ & $0.90 \pm 0.03$ & $0.90 \pm 0.03$ \\
Arg188 & $0.93 \pm 0.12$ & $0.89 \pm 0.01$ & $0.76 \pm 0.23$ \\
\hline
\end{tabular}

3 